\title{
Distinct effects of different adjuvants in the mouse model of allergic airway inflammation
}

\author{
Hyun Soo Shin ${ }^{1,2 *}$ Hye Rin Chun, ${ }^{3 *}$ Hye Young Na, ${ }^{1}$ Moah Sohn ${ }^{1,2}$ Seul Hye Ryu, ${ }^{1,2}$ Wanho Choi, ${ }^{1,2}$ \\ Hyunju In, ${ }^{1,2}$ Ji Soo Park, ${ }^{1,2}$ Sejung Park, ${ }^{1,2}$ Chae Gyu Park ${ }^{1,2,4}$
}

\begin{abstract}
Background: Allergic asthma was typically considered as an inflammatory disease mediated by type 2 immunity. However, recent studies revealed that asthma is a complex disease displaying a variety of phenotypes and endotypes.

Objective: We examined cellular phenotypes in the mouse model of allergic asthma sensitized with different adjuvants. The aim of our study was to determine immunologic cellular characteristics in mouse asthma models induced by ovalbumin (OVA) and a variety of adjuvants.
\end{abstract}

Method: Mice were sensitized intraperitoneally with the admixture of OVA and various adjuvants such as Alhydrogel (alum), papain, lipopolysaccharide (LPS), or CpG, and subsequently challenged with OVA intranasally. The cells in bronchoalveolar lavage (BAL) fluid, lung, and mediastinal lymph node $(\mathrm{mLN})$ were examined by flow cytometric analyses.

Results: In the lung and BAL fluid, the highest eosinophil levels were observed in the alum group while the highest neutrophil levels were detected in the LPS group. Meanwhile, the LPS group exhibited the most elevated levels of both ROR $\mathrm{t}^{+}$innate lymphoid cells (ILCs) and IL-17A $\mathrm{A}^{+}$Th cells in the lung and mediastinal lymph node. In the lung, the number of T-bet ${ }^{+}$ILCs was highest in the papain group whereas the number of IFN- $\gamma^{+}$Th cells was highest in the CpG group.

Conclusion: Notable variances are found in the composition of immune cells and expression of cytokines at the site of pathogenesis among the different mouse models of allergic asthma created by the sensitization with different adjuvants.

Key words: Adjuvants, Allergy, Asthma, Inflammation, Lung

\section{Citation:}

Shin, H. S., Chun, H. R., Na, H. Y., Sohn, M., Ryu, S. H., Choi, W., In, H., Park, J. S., Park, S., Park, C. G. (2022). Distinct effects of different adjuvants in the mouse model of allergic airway inflammation. Asian Pac J Allergy Immunol, 40(2), 111-120. https:// doi.org/10.12932/ap-301219-0729

\section{Affiliations:}

${ }^{1}$ Laboratory of Immunology, Severance Biomedical Science Institute, Yonsei University College of Medicine, Seoul, Republic of Korea

${ }^{2}$ Brain Korea 21 PLUS Project for Medical Science,

Yonsei University College of Medicine, Seoul, Republic of Korea

${ }^{3}$ Integrated Science and Engineering Division, Underwood

International College, Yonsei University, Seoul, Republic of Korea

${ }^{4}$ Institute for Immunology and Immunological Diseases,

Yonsei University College of Medicine, Seoul, Republic of Korea

\section{Corresponding author:}

Chae Gyu Park

Laboratory of Immunology, Severance Biomedical Science Institute,

Brain Korea 21 PLUS Project for Medical Science,

Yonsei University College of Medicine,

50-1 Yonsei-ro, Seodaemun-gu, Seoul 03722, Republic of Korea

E-mail: ChaeGyu@yuhs.ac

* Contributed equally

\section{Introduction}

Allergic asthma is an airway inflammatory disease characterized with airway hyperresponsiveness (AHR), eosinophilic inflammation, and reversible airway obstruction. ${ }^{1}$ Classically, allergic asthma was considered a disease mediated by $\mathrm{T}$ helper 2 (Th2) cells. This is based on the observations that allergic symptoms are often associated with the airway infiltration of inflammatory cells, most notably eosinophils, $\mathrm{CD} 4^{+} \mathrm{T}$ cells, and mast cells. Also, Th2 cytokines, like IL-4, IL-5, and IL-13, 
are expressed by infiltrating $\mathrm{CD} 4^{+} \mathrm{T}$ cells in the sites of pathogenesis. ${ }^{2}$ However, recent evidences have suggested that allergic asthma is a heterogeneous disease. In addition to Th2 cells, Th17 cells are indicated to involve in complicated allergic asthma. Th17 cells are found in AHR, steroid resistance, and neutrophilic inflammation, ${ }^{3,4}$ where the severity of disease correlates with Th17 cell activity and neutrophil recruitment. ${ }^{5,6}$

Innate lymphoid cells (ILCs), recently discovered lymphoid effector cells, consist of 3 functionally distinct groups, i.e., ILC1s, ILC2s, and ILC3s. The relationship between ILC2s that produce Th2 cytokines and asthmatic inflammation is well documented. When sensitized with allergen and adjuvant papain, ILC2s prompted an eosinophilic inflammation in RAG2-deficient mice. ${ }^{7}$ Besides, mice lacking ILC2s were shown to exhibit the compromised responses of Th2 cells to allergens, underlining the crosstalk between ILC2s and T cells. ${ }^{8}$ IL-17A-producing ILC3s have been observed in obese mice that spontaneously developed AHR and in the bronchoalveolar lavage (BAL) fluid of asthma patients. ${ }^{9}$ Similarly, the number of ILC3s increased in the BAL fluid from mice challenged with organic swine barn dust extract. ${ }^{10}$

Although allergic asthma has been recognized to possess complex characteristics of airway inflammation, it is not clear which mouse models can appropriately represent the diverse features of this complicated disease. In the present study, therefore, we evaluated the mouse models of allergic airway inflammation sensitized with different adjuvants. We found that the use of different adjuvants in sensitization protocol produced distinct cellular composition and cytokine expression at the site of pathogenesis in the mouse models of allergic airway inflammation.

\section{Materials and Methods Animals}

Female BALB/c mice were purchased from Orient Bio (Seongnam, Republic of Korea). Mice were maintained in specific pathogen-free facilities of the Department of Laboratory Animal Resources at the Yonsei University College of Medicine. Mice were used at 8 to 12 weeks of age in all experiments. Animal care and experiments were carried out humanely according to the guidelines and protocol (\#2017-0001) approved by the Institutional Animal Care and Use Committees of the Yonsei University College of Medicine.

\section{Antibodies and Reagents}

Fluorochrome-conjugated monoclonal antibodies (mAbs) were purchased from BioLegend (San Diego, CA): PE-Cy7conjugated anti-CD3 (clone 17A2), anti-CD19 (clone 1D3/ CD19), anti-CD49b (clone DX5), anti-IL-4 (clone 11B11); Alexa Flour 488-conjugated anti-CD45.2 (clone 104), antiTCR- $\beta$ chain (clone H57-597); Brilliant Violet 421-conjugated anti-CD11b (clone M1/70), anti-CD8 (clone 53-6.7), antiGATA3 (clone 16E10A23); APC-Cy7-conjugated anti-I-A/I-E (anti MHC class II, clone M5/114.15.2), anti-IL-7Ra (clone A7R34), anti-IL-17A (clone TC11-18H10.1); PerCP/Cy5.5conjugated anti-Ly6G (clone 1A8), anti-CD3 (clone 17A2), anti-CD11b (clone M1/70), anti-IFN- $\gamma$ (clone XMG1.2);
APC-conjugated anti-CD3 (clone 17A2), anti-T-bet (clone 4B10), anti-IL-5 (clone TFRK5); PE-conjugated anti-CD11c (clone N418), anti-CD4 (clone GK1.5). Isotype control mAbs were also purchased from BioLegend: PE-Cy7-, APC-, APCCy7-, PE-, and PerCP/Cy5.5-conjugated rat IgG1/ $\kappa$ isotype control mAb (clone RTK2071); APC-conjugated mouse Ig$\mathrm{G} 1 / \kappa$ isotype control $\mathrm{mAb}$ (clone MOPC-21); Brilliant Violet 421-conjugated mouse $\operatorname{IgG} 2 \mathrm{~b} / \mathrm{\kappa}$ isotype control $\mathrm{mAb}$ (clone 27-35); and PerCP/Cy5.5-conjugated Armenian hamster IgG isotype control mAb (clone HTK888). Alexa Flour 647-conjugated anti-SiglecF (clone E50-2440) was purchased from BD Biosciences (San Jose, CA) and PE-conjugated anti-ROR $\gamma \mathrm{t}$ (clone AFKJS-9) from Thermo Fisher Scientific (Waltham, MA). LIVE/DEAD fixable dead cell stain kit was purchased from Thermo Fisher Scientific.

\section{Mouse models of allergic airway inflammation and cell iso- lation}

Mice were sensitized by intraperitoneal (i.p.) administration of $100 \mu \mathrm{g}^{11-14}$ of soluble ovalbumin (OVA, Grade V, Sigma-Aldrich, St. Louis, MO) $)^{15-18}$ and, after $30 \mathrm{~min}$, by another i.p. injection of control PBS or an adjuvant listed as follows: $200 \mu \mathrm{g}$ of Alhydrogel (alum; InvivoGen, San Diego, CA), ${ }^{19}$ $100 \mu \mathrm{g}$ of papain (Sigma-Aldrich), ${ }^{20} 10 \mu \mathrm{g}$ of LPS (Sigma-Aldrich), ${ }^{14,21}$ or $50 \mu \mathrm{g}$ of $\mathrm{CpG}$ (Enzo Life Sciences, Farmingdale, $\mathrm{NY}){ }^{22}$ For allergen challenge, $100 \mu \mathrm{g}$ of OVA in $20 \mu \mathrm{l}$ PBS was administrated intranasally (i.n.) once a day for 5 days (from day 10 to day 14 after sensitization). ${ }^{23}$ All mice in experiments were sacrificed for analysis at day 15 (Figure 1). Each mouse was euthanized by $\mathrm{CO}_{2}$ and BAL fluid was collected with $1 \mathrm{ml}$ HBSS without calcium and magnesium. On the same day, mediastinal LNs (mLNs) were harvested and single cell suspensions were prepared by homogenizing through $100 \mu \mathrm{m}$ cell strainers before wash and resuspension in HBSS. For analysis of the lung, mice were perfused gently with a syringe containing $10 \mathrm{ml}$ of HBSS prior to harvesting lungs. After incubation at $37^{\circ} \mathrm{C}$ for $30 \mathrm{~min}$ in collagenase D (Roche, Basel, Switzerland) followed by treatment of $20 \mathrm{mM}$ of EDTA for $5 \mathrm{~min}$, single cell suspensions were prepared by homogenizing lung tissues through $100 \mu \mathrm{m}$ cell strainers. Then, cells from lung tissues were subjected to lysis of erythrocytes with ACK lysis buffer (BioLegend) before wash and resuspension in HBSS.

\section{Flow cytometry}

Flow cytometric analyses were carried out as described previously. ${ }^{24-27}$ In brief, samples of single cell suspension from BAL fluid, mLN, and lung were treated with an Fc blocking reagent containing the culture supernatant of $2.4 \mathrm{G} 2$ (anti$\mathrm{CD} 16 / 32) \mathrm{mAb}$ hybridoma for $20 \mathrm{~min}$ at $4^{\circ} \mathrm{C}$. Then, the samples were washed with FACS buffer (2\% fetal calf serum, 2 mM EDTA, $0.1 \%$ sodium azide) followed by incubation with the mixture of fluorochrome-conjugated mAbs and live/dead dye for $20 \mathrm{~min}$ at $4^{\circ} \mathrm{C}$. After wash with FACS buffer, each sample was analyzed with FACSVerse ${ }^{\mathrm{Tm}}$ flow cytometer (BD Biosciences, San Jose, CA). The mixture of anti-lineage (Lin) markers consisting of anti-CD3, anti-CD19, and anti-CD49b was used to exclude T cells, B cells, and NK cells prior to gating on eosinophils, neutrophils, and dendritic cells (DCs). 


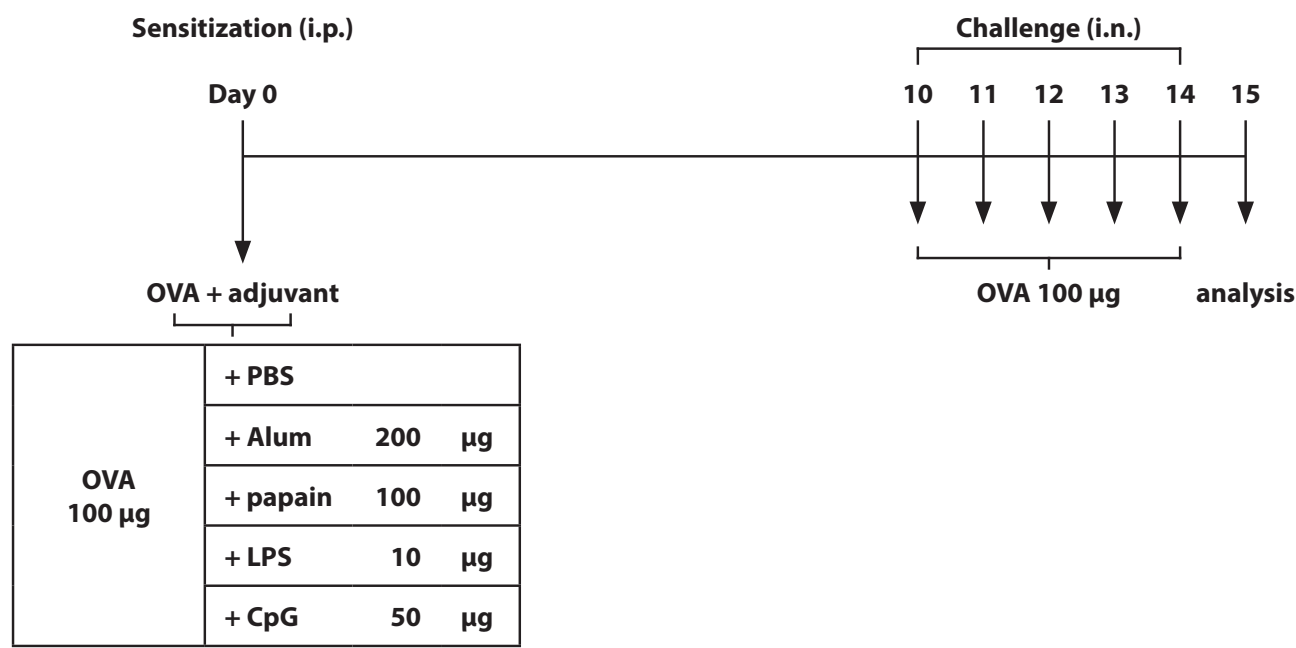

Figure 1. Schematic view of sensitization and challenge protocols for allergic airway inflammation.

Eosinophils were defined as $\mathrm{CD}_{4} .2^{+} \mathrm{Lin}^{-\mathrm{CD}} 11 \mathrm{~b}^{+}$SiglecF $^{+}$ Ly6G; neutrophils as $\mathrm{CD}^{-} 45.2^{+} \mathrm{Lin}^{-\mathrm{CD} 11 \mathrm{~b}^{+} \text {SiglecF-Ly6G }}{ }^{+}$; DCs

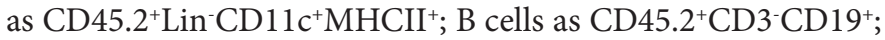
Th cells as $\mathrm{CD} 45.2^{+} \mathrm{CD}^{+} \mathrm{CD}^{+}$; and cytotoxic $\mathrm{T}(\mathrm{Tc})$ cells as $\mathrm{CD} 45.2^{+} \mathrm{CD}^{+} \mathrm{CD}^{+}$. The gating strategies for ILC subsets are described previously. ${ }^{28}$ In brief, each sample was first labeled on surface with the mixture of anti-CD45.2, anti-IL7Ra, and anti-Lin markers (anti-CD3, anti-CD5, anit-CD11b, antiCD11c, anti-B220); and then intracellularly stained with the mixture of anti-CD3, anti-GATA3, anti-T-bet, and anti-ROR $\gamma \mathrm{t}$ using True-NuclearTM Transcription Factor Staining reagents and protocol (BioLegend). ILCs were classified into T-bet ${ }^{+}$ ILC1s, GATA3 ${ }^{+}$ILC2s, and ROR $\gamma \mathrm{t}^{+}$ILC3s after gating on CD45.2 ${ }^{+} \mathrm{Lin}^{-} \mathrm{IL}-7 \mathrm{Ra}^{+}$.

\section{Intracellular cytokine assay}

Intracellular cytokine assay was performed as described previously. ${ }^{29}$ In brief, each sample of single cell suspension was treated with PMA $(12 \mathrm{nM})$, ionomycin $(1 \mu \mathrm{M})$, and Brefeldin A $(5 \mu \mathrm{g} / \mathrm{ml})$ at $37^{\circ} \mathrm{C}$ for 4 hours. After treatment, the sample was incubated with Fc blocking 2.4G2 as described above. Then, the sample was labeled on surface with the mixture of anti-TCR $\beta$, anti-CD4, and anti-CD8, followed by intracellular staining with the mAb cocktail containing anti-IL-4, anti-IL-5, anti-IL-17A, and anti-IFN- $\gamma$ using Fixation/Permeabilization solution (BioLegend).

\section{Statistics}

Group-averaged data were analyzed by one-way ANOVA and plotted using Prism 5 (GraphPad Software, La Jolla, CA). Statistical significance is indicated by the $p$ values below 0.05 $\left.{ }^{*}\right), 0.01\left(^{* *}\right)$, and $0.001\left(^{* *}\right)$.

\section{Results}

Distinct changes in immune cell populations are observed between BAL fluids from allergic airway inflammations induced with different adjuvants

To evaluate the populations of various immune cells in distinct models of allergic asthma, BALB/c mice were sensitized i.p. with OVA in combination with a different adjuvant such as alum, papain, LPS, and CpG. After 10 days, mice were challenged i.n. with OVA once a day for 5 days before sacrificed (Figure 1). Then, we analyzed those mice by assessing the numbers of eosinophils, neutrophils, DCs, Th, Tc, and B cells in BAL fluids and lungs. As shown in Figure 2a, the BAL fluids from mice sensitized using allergen OVA without adjuvant, i.e. OVA with PBS, contained much higher numbers of immune cell populations than those from control mice without sensitization and challenge. In BAL fluids among mice sensitized with a different adjuvant or PBS (Figure $2 \mathbf{b}$ ), the group sensitized with alum exhibited the highest percentage of eosinophils whereas those treated with LPS and CpG showed the lowest. The population of neutrophils in BAL fluids was significantly augmented in the groups sensitized with LPS or CpG. B cell population in the alum group increased markedly compared to the PBS group, while the populations of DCs, Th, and Tc cells displayed no significant changes between different adjuvant groups.

Population changes of eosinophils and neutrophils in lungs between mice sensitization with different adjuvants are comparable to those in BAL fluids

From mice sensitized with different adjuvants, after collecting BAL fluids, single cell suspensions of lung tissues were prepared for analysis by flow cytometry. As illustrated in Figure 3a, lungs from mice sensitized and challenged with allergen OVA exhibited higher levels of eosinophils and DCs than those from control mice without sensitization and challenge. In lungs among mice sensitized with a different adjuvant or PBS (Figure 3b), the alum group maintained the highest levels of eosinophils whereas the LPS and CpG groups had the lowest. Meanwhile, compared to the PBS group, the population of neutrophils increased significantly in the LPS group while those of DCs and B cells were significantly augmented in the CpG group. For T cells, no significant differences were noticed in Th cells but the percentage of Tc cells was reduced in the lung from the alum and $\mathrm{CpG}$ groups. By and large, the populations of eosinophils and neutrophils in the lung have changed similarly to those in BAL fluids of mice sensitized with different adjuvants. 
(a)
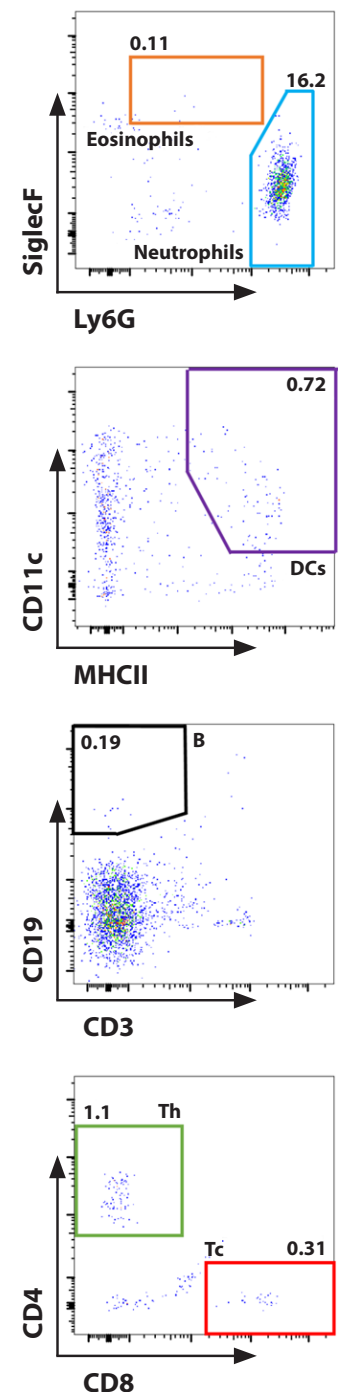
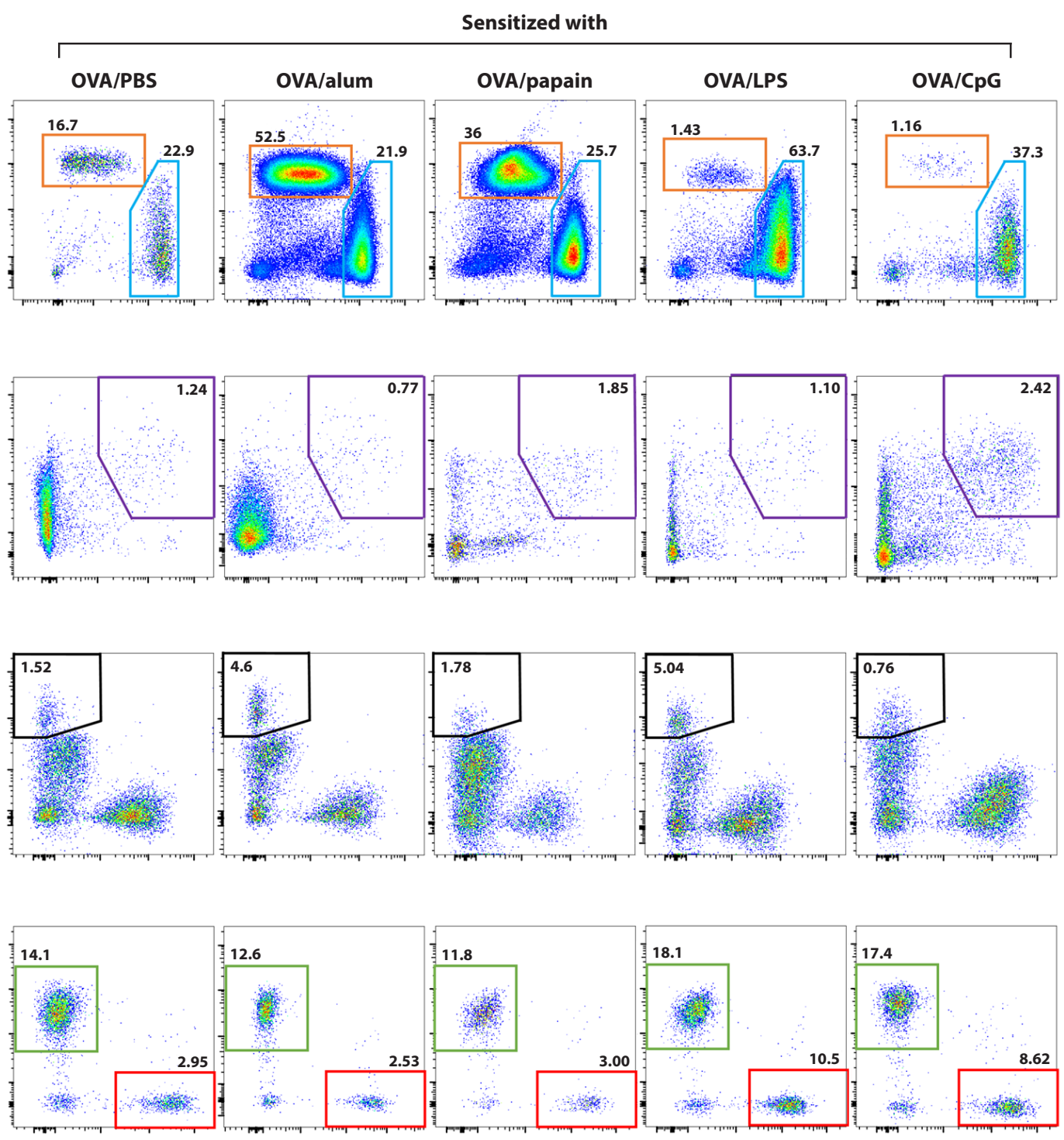

(b)

Eosinophils

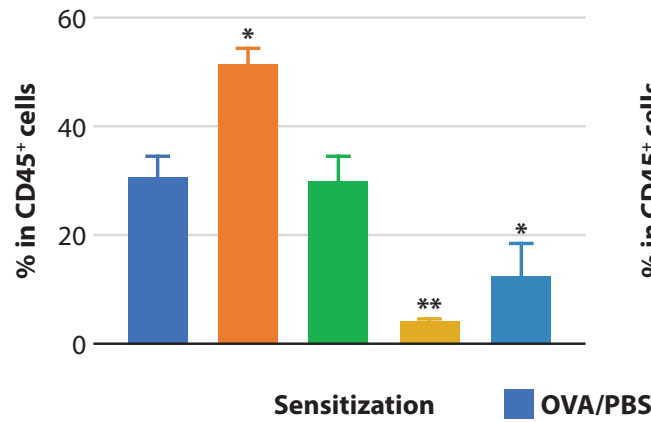

Neutrophils

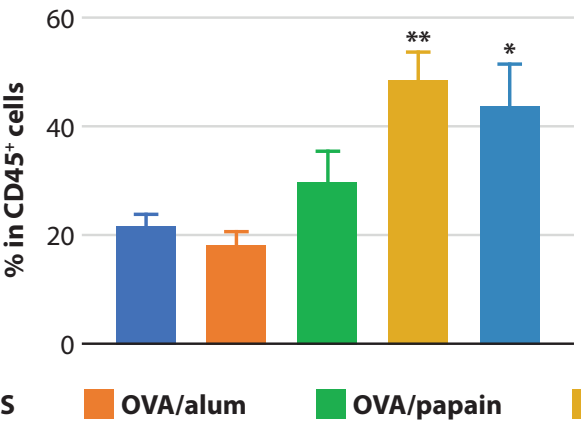

DCs

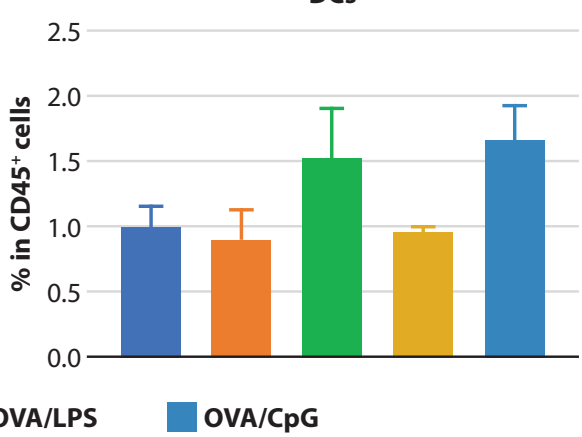

Figure 2. Flow cytometric analysis on the levels of immune cells in BAL fluids from the mice sensitized i.p. with OVA and different adjuvants and challenged i.n. with OVA as in Figure 1. CD45 ${ }^{+}$cells in BAL fluids are used to identify immune cells. (a) Representative flow cytograms are illustrated with gating strategies used to identify each cell type in BAL fluids on the basis of indicative surface markers: Lin (CD3, CD19, CD49b), Eosinophils (Lin-, CD11b ${ }^{+}, \mathrm{CD}^{-} 1 \mathrm{c}^{-}$, SiglecF ${ }^{+}$), Neutrophils (Lin-, CD11b

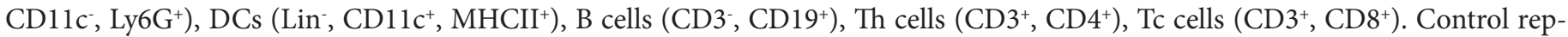
resents a result from mice without sensitization and challenge. (b) Histograms depict the levels of total eosinophils, neutrophils, DCs, B cells, Th cells, and Tc cells in BAL fluids from mice sensitized and challenged as in Figure $1 .{ }^{*} p<0.05,{ }^{* *} p<0.01,{ }^{* *} p$ $<0.001$, compared with the PBS (OVA without adjuvant but PBS) group; $\mathrm{n} \geq 7$. 
(b) (Continued)

B cells

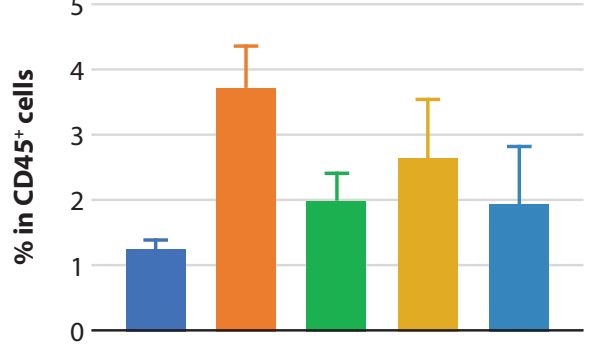

Sensitization

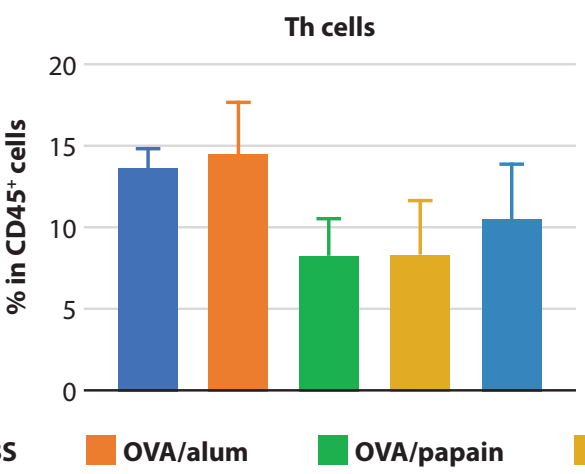

Tc cells

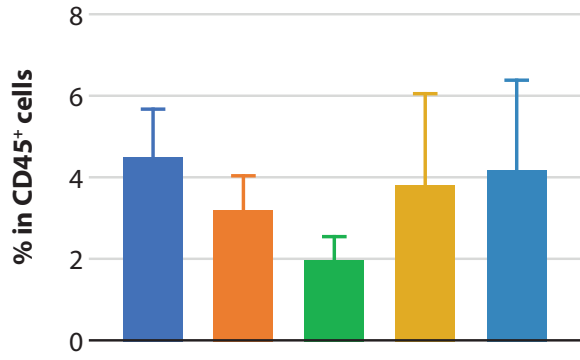

OVA/LPS OVA/CPG

Figure 2. (Continued)

(a)
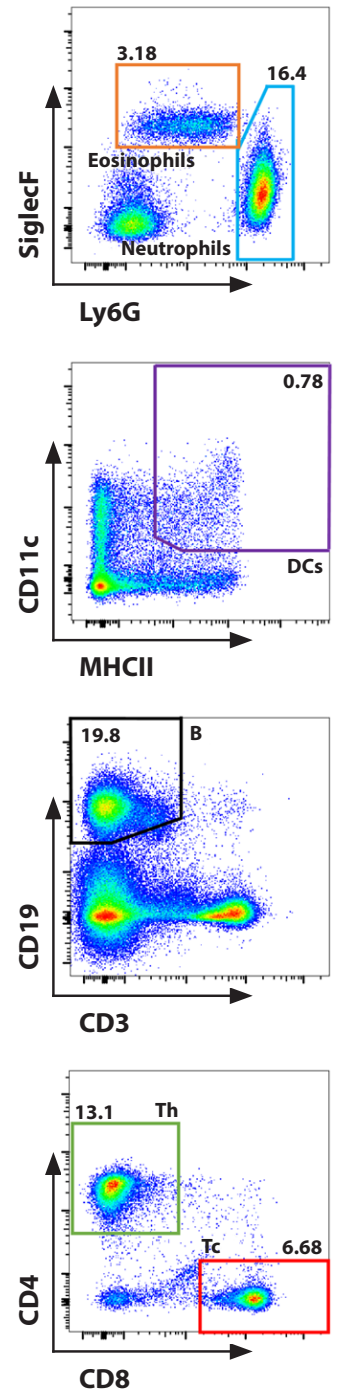

Sensitized with
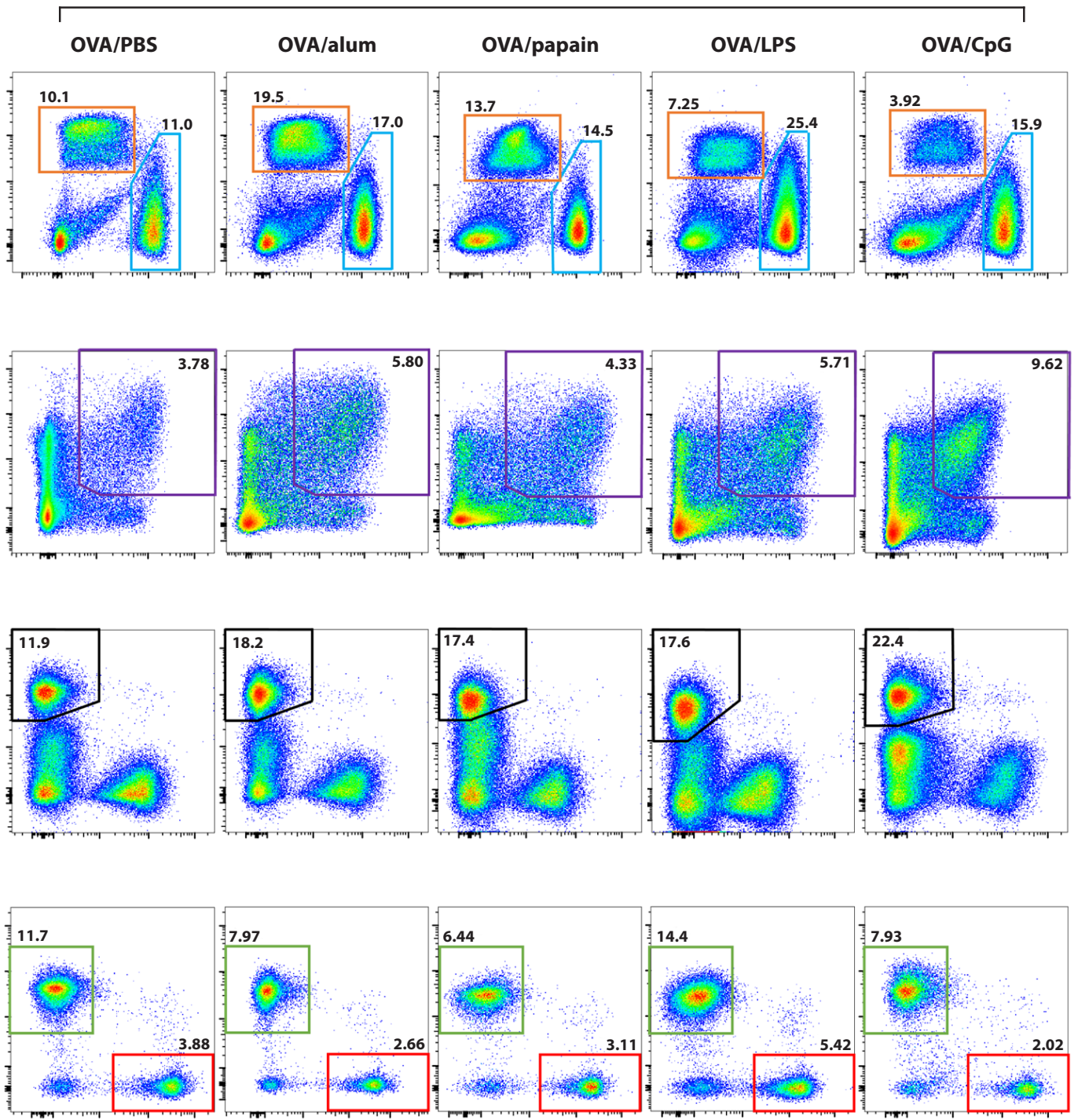

Figure 3. Flow cytometric analysis on the levels of immune cells in lungs from the mice sensitized i.p. with OVA and different adjuvants and challenged i.n. with OVA as in Figure 1. (a) Representative flow cytograms are shown with gating strategies used to identify each cell type in lung tissues as in Figure 2. (b) Histograms display the levels of each type of immune cells in lungs from the respective groups. ${ }^{*} p<0.05,{ }^{* *} p<0.01,{ }^{* * *} p<0.001$, compared with the PBS group; $\mathrm{n} \geq 7$. 
(b)
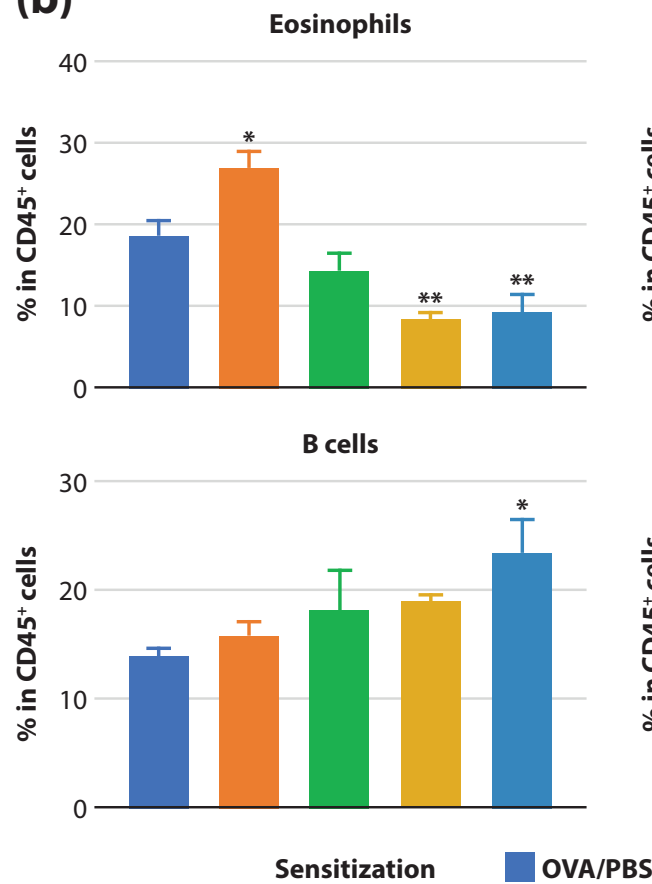

Figure 3. (Continued)

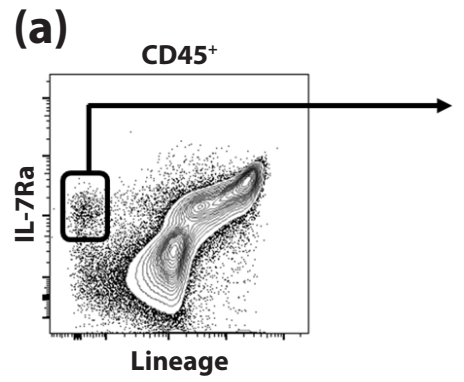

Lineage

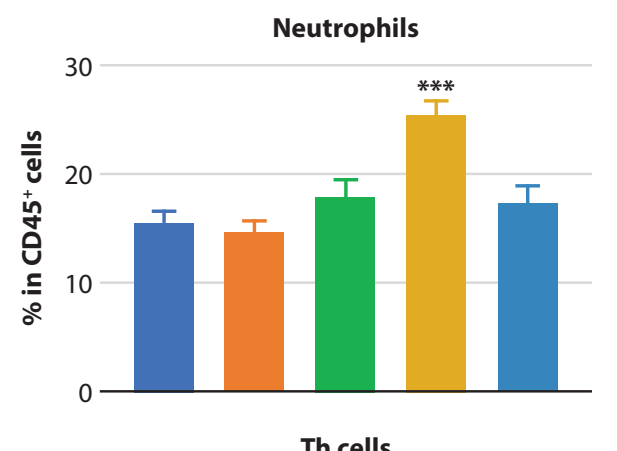

Th cells

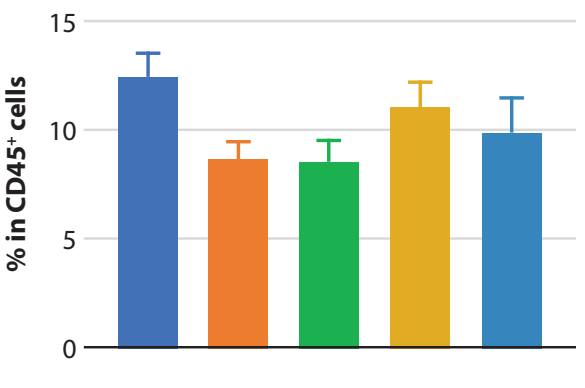

OVA/alum

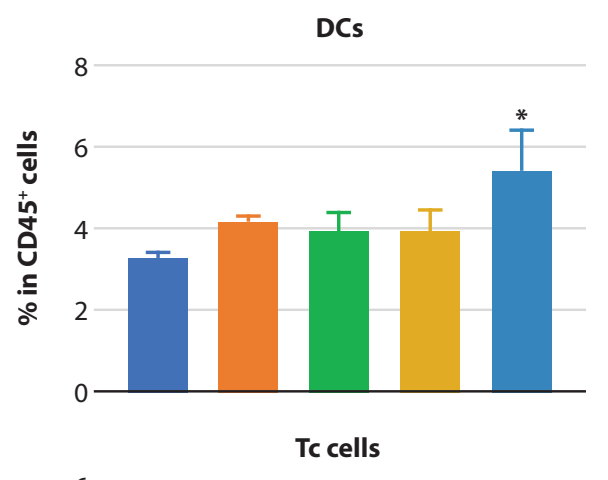

6

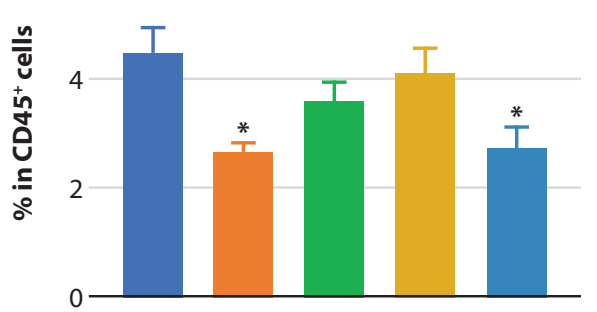

OVA/LPS OVA/CPG
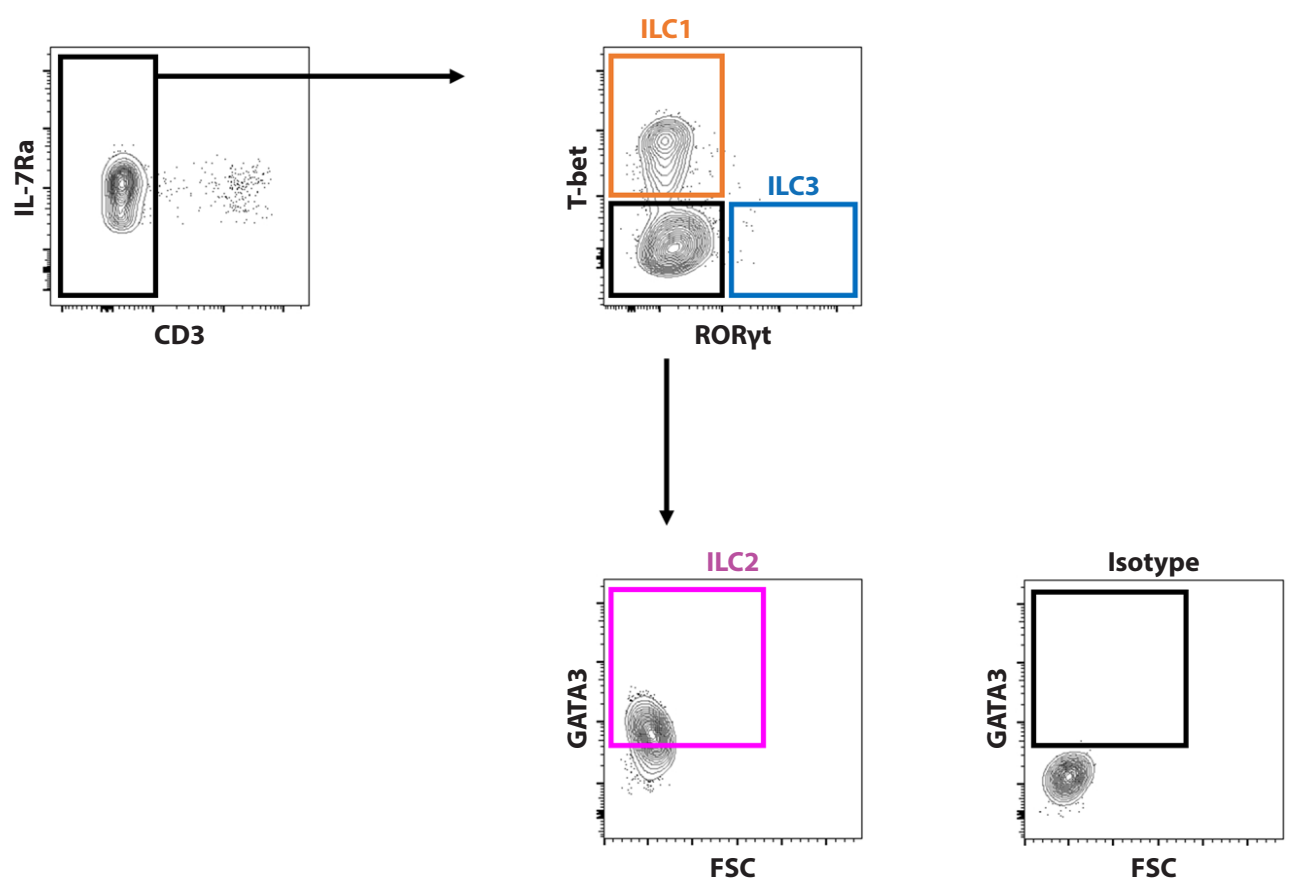

Figure 4. Histograms illustrate the levels of ILCs in tissues from the mice sensitized and challenged as in Figure 1. (a) The flow cytometry gating strategies for analyzing ILC subsets. $\mathrm{CD}_{4} 5^{+}$cells in lungs (b) and mLNs (c) from mice in each group are analyzed flow cytometrically on the basis of indicative surface and intracellular markers for ILCs: Lin (CD3, CD5, CD11b, CD11c, B220), ILC1s (Lin', IL7Ra ${ }^{+}$, intracellular CD3- and T-bet ${ }^{+}$), ILC2s (Lin-, IL7Ra ${ }^{+}$, intracellular CD3- and GATA3 ${ }^{+}$), ILC3s (Lin-, IL7Rat, intracellular CD3 and ROR $\left.\gamma \mathrm{t}^{+}\right) .{ }^{*} p<0.05,{ }^{* *} p<0.01,{ }^{* * *} p<0.001$, compared with the PBS group; $\mathrm{n} \geq 3$. 


\section{(b) Lung}

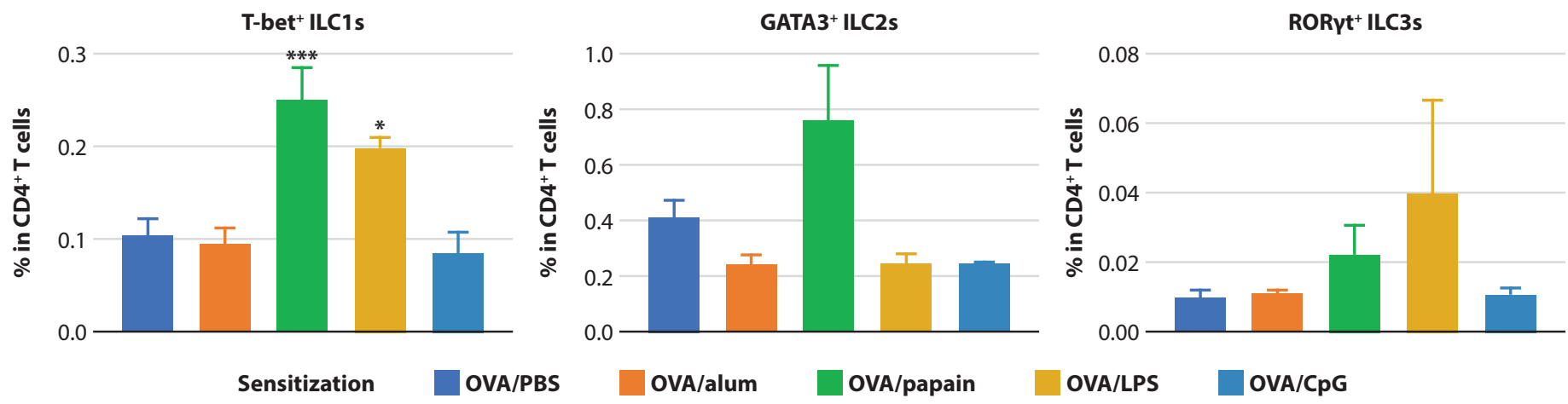

\section{(c) $\mathrm{mLN}$}
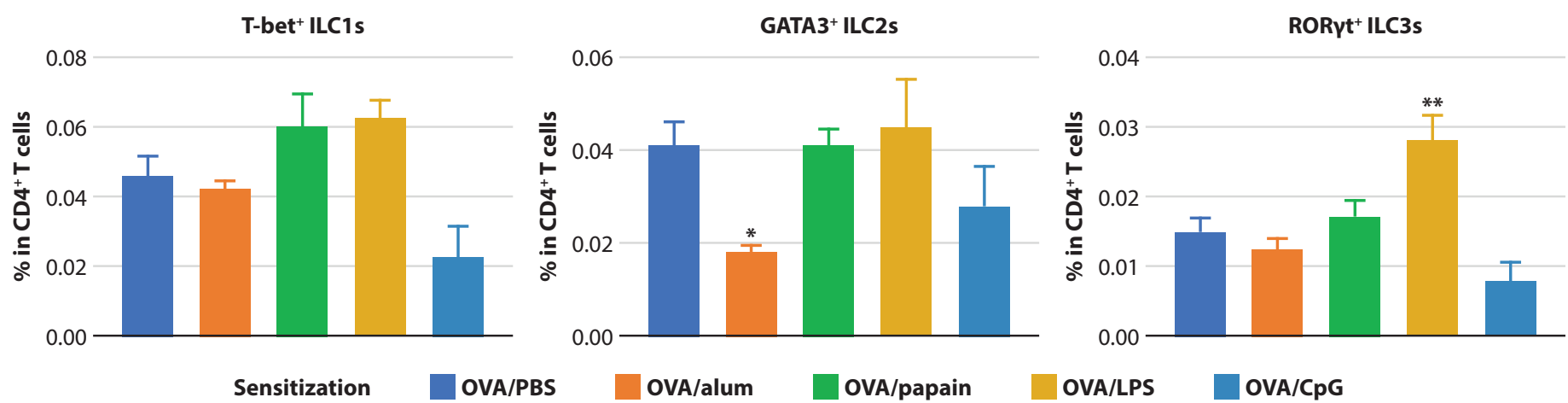

Figure 4. (Continued)

(a) Lung

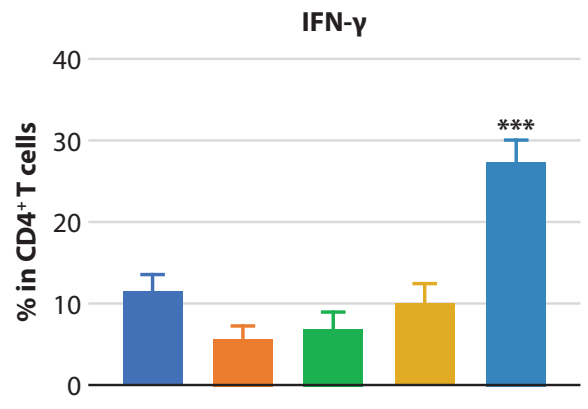

IL-5

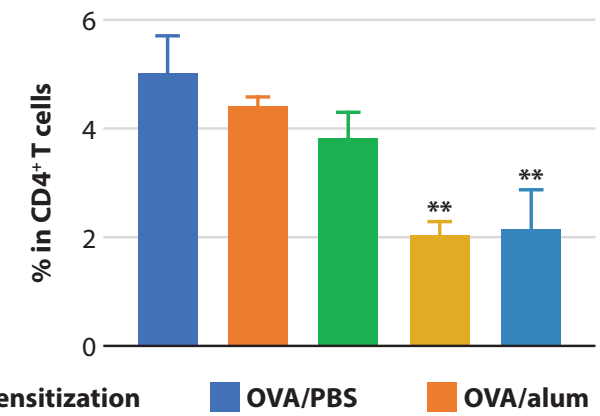

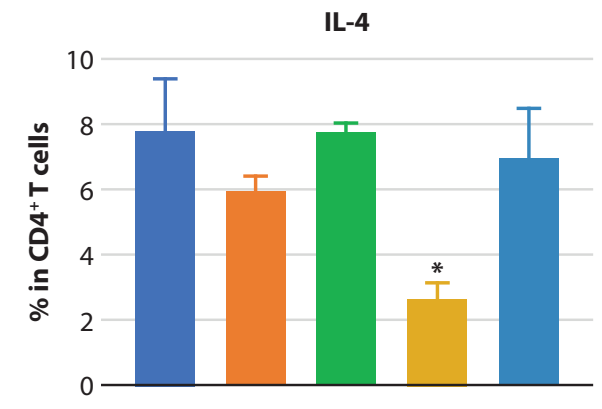

IL-17A

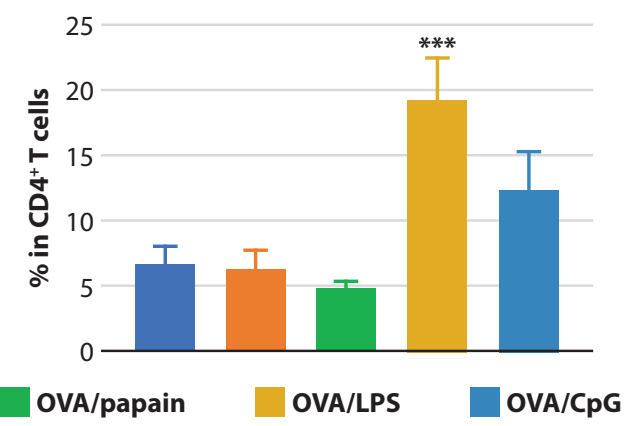

Figure 5. Histograms display the levels of cytokines in tissues from the mice sensitized and challenged as in Figure 1. Following stimulation with PMA/ionomycin, $\mathrm{CD}^{+}$Th cells in lungs (a) and mLNs (b) from mice in each group are analyzed flow cytometrically to evaluate intracellular cytokines. ${ }^{*} p<0.05,{ }^{* *} p<0.01,{ }^{* *} p<0.001$, compared with the PBS group; $\mathrm{n} \geq 5$. 
(b) $\mathrm{mLN}$

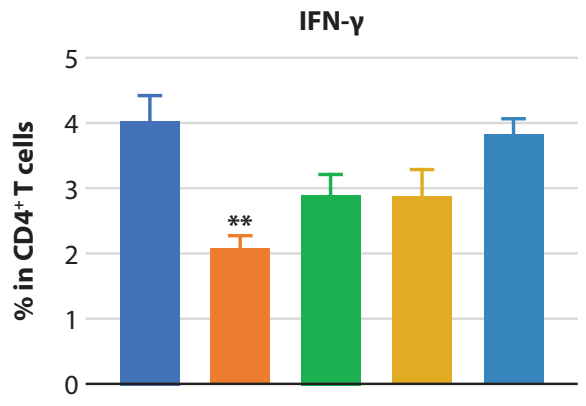

IL-5

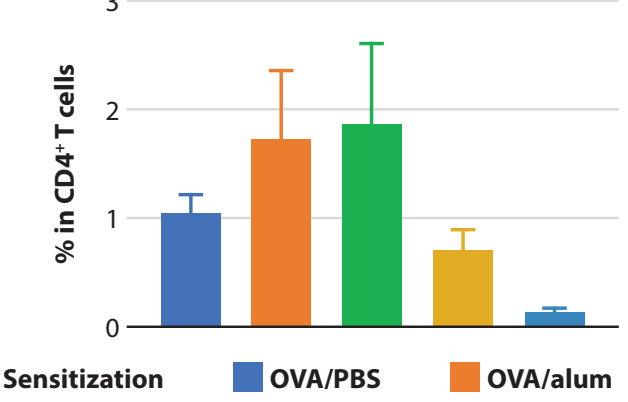

Figure 5. (Continued)

Distinct changes in ILC subsets are detected in lungs and mLNs from mice sensitized with different adjuvants

Since numerous studies on the involvement of ILCs in allergic asthma have been recently reported, ${ }^{30}$ we investigated to see whether the populations of three ILC subtypes would be affected by the sensitization with different adjuvants. Each of three ILC subsets was identified as T-bet ${ }^{+}$for ILC1s; as $\mathrm{GATA}^{+}$for ILC2s; and as ROR $\gamma \mathrm{t}^{+}$for ILC3s from Lin IL-7 $\mathrm{Ra}^{+}$hematopoietic cells in the lung and mLN. In lungs (Figure 4b), the papain and LPS groups showed the significant increase of T-bet ${ }^{+}$ILC1s. The papain group also exhibited the highest percentage of GATA3 ${ }^{+}$ILC2s in the lung. In mLNs (Figure 4c), the CpG group showed the lowest levels of T-bet ILC1s; the alum group had the lowest levels of GATA $^{+}$ILC2s; and the LPS group exhibited the highest levels of ROR $\gamma \mathrm{t}^{+}$ILC3s.

Th cells in lung and mLN exhibit the distinct pattern of cytokine expression between different adjuvant groups.

During asthma exacerbation, not only Th2 and Th17 related cytokines but also the level of IFN- $\gamma$ associated with Th1 are increased..$^{31}$ Therefore, we evaluated $\mathrm{CD}^{+}{ }^{+}$Th cells in the lung and mLN from different adjuvant groups for their intracellular expression of four distinct cytokines, i.e., IFN- $\gamma$ (Th1); IL-4, IL-5 (Th2); and IL-17A (Th17) following stimulation using PMA and ionomycin with Brefeldin A. In lung tissues (Figure 5a), the CpG group exhibited much higher levels of IFN- $\gamma^{+}$Th1 cells than the other adjuvant groups. Both the LPS and CpG groups possessed statistically the lowest percentages of IL- $5^{+}$Th2 cells in the lung, while the LPS group also had the lowest levels of IL- $4^{+}$Th2 cells. In contrast to Th2 cells, the LPS group displayed a significant increase of IL-17A ${ }^{+}$Th17 cells and likewise the CpG group showed some increase of IL-17A ${ }^{+}$Th17 cells in lungs. In mLNs (Figure 5b), the population of IFN- $\gamma^{+}$Th1 cells was statically smaller

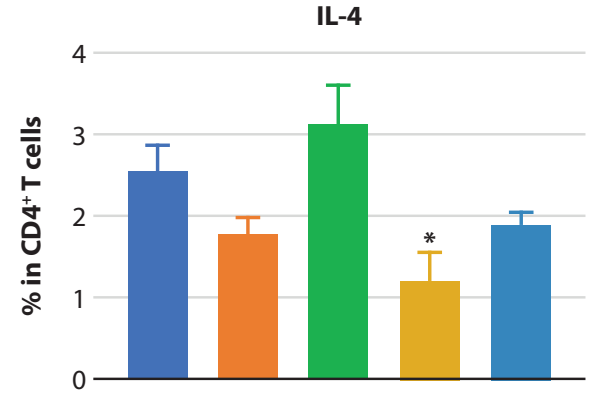

IL-17A

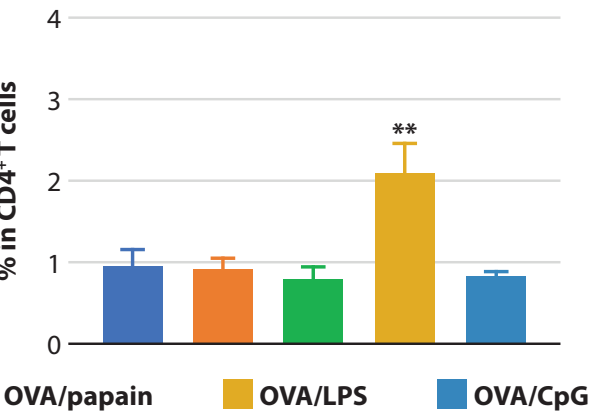

in the alum group than in the others. Somewhat similar to the results of lungs, the percentage of IL- $4^{+}$Th2 cells was the lowest in the LPS group and that of IL- $5^{+}$Th2 cells was the lowest in the CpG group in mLNs. The levels of IL-17A+ Th17 cells in mLNs were detected much higher in the LPS group than in the other adjuvant and PBS groups as observed similarly in lungs.

\section{Discussion}

Allergic asthma is a complex inflammatory disease of the airways accompanied by infiltration of inflammatory cells causing AHR, mucus overproduction, and airway remodeling. ${ }^{32}$ Once thought as a Th2 cell-mediated disease, it is now widely considered heterogeneous in nature with many endotypes. Various endotypes of allergic asthma have been studied, including the Th2 cell-, Th17 cell-, ILC2-mediated forms and more, but a definitive baseline for this disease's cellular and cytokine characteristics is yet to be established..$^{33}$ In the present study, we created 5 different OVA-sensitized airway inflammation models using PBS and 4 different adjuvants, including alum, papain, LPS, and CpG, and investigated variances in the profiles of immune cells and cytokines among the

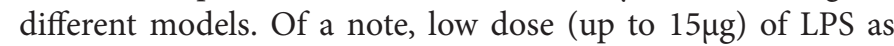
an adjuvant generate boosting effects in allergic response, ${ }^{14,34,35}$ while high dose (50 to $100 \mu \mathrm{g}$ ) of LPS inhibit HDM-induced allergic asthma as well as food allergy. ${ }^{34,35}$

In the mice sensitized with OVA and alum followed by challenge of OVA, allergic airway inflammation exhibited the characteristics of a traditional Th2 cytokine-mediated eosinophilic inflammation in agreement with the previous studies. ${ }^{1}$ Meanwhile, the group sensitized with papain revealed increases in the populations of T-bet ${ }^{+}$ILC1s and GATA3 ${ }^{+}$ ILC2s in the lung, in line with the previous finding that ILC2s are activated by papain and Alternaria alternate through an event in the lung, ${ }^{7}$ but indicating an endotype different 
from that of the alum group. In the LPS group, characteristic increases in the populations of neutrophils, ROR $\gamma \mathrm{t}^{+}$ILC3s, and IL- $17 \mathrm{~A}^{+}$Th17 cells resembled a typical Th-17 cytokine-mediated neutrophilic inflammation. Similar to the LPS group, the mouse model sensitized with CpG displayed neutrophilic phenotypes in BAL fluids, but not in the lung, with the highest levels of DCs and B cells in the lung. Though the decreased levels of IL- $5^{+}$Th2 cells were detected in both CpG and LPS groups, the reduced levels of IL- $4^{+}$Th2 cells were observed only in the LPS group. Besides, the augmented levels of IFN- $\gamma^{+}$Th1 cells were observed only in the lung of the CpG group. We speculate that this distinct endotype of the $\mathrm{CpG}$ and LPS groups might explain the therapeutic role of $\mathrm{CpG}$ and LPS in allergic asthma. ${ }^{36,37}$ It is noticeable that in the lung of the CpG group, the percentage of T-bet ${ }^{+}$ILC1s did not changed, while that of IFN $-\gamma^{+}$Th1 cells increased markedly. Therefore, it will be interesting to evaluate the level of IFN- $\gamma$ expression in T-bet ${ }^{+}$ILC1s and that of T-bet expression in IFN- $\gamma^{+}$Th1 cells from the lung of the CpG group. In addition, the examination of the respective levels of T-bet, GATA3, and ROR $\gamma \mathrm{t}$ in IFN- $\gamma^{+}$, IL- $4^{+}$, IL- $5^{+}$, and IL- $17 \mathrm{~A}^{+}$Th cells might provide further information on the heterogenous endotypes generated by the treatment of distinct adjuvants.

By sensitizing mice with allergen OVA and different adjuvants we could generate experimental mouse models of allergic airway inflammation with distinct endotypes. Understanding the heterogeneity of allergic asthma and gaining the ability to generate appropriate mouse models may further our effort to develop more effective therapies that are tailored to specific cell types and pathophysiological mechanisms. Our study indicates that further investigations are required with many other types of allergens and adjuvants as well as immune cells and cytokines. Besides, we did not evaluate the IL-13 ${ }^{+}$Th cells, although the level of IL-13 was known to be related with the severe asthma. ${ }^{38}$ Nevertheless, our present study lays the groundwork into making allergic airway inflammation models with complex immunological endotypes.

\section{Acknowledgements}

We thank dedicated services provided by the Flow Cytometry Core of the Yonsei Biomedical Research Institute and the Department of Laboratory Animal Resources at the Yonsei University College of Medicine. This work was supported by grants from the National Research Foundation of Korea to CGP (NRF-2014R1A4A1008625, NRF-2017M3A9C8064887, NRF-2019R1F1A1041700) and HYN (NRF-2017R1A6A3A11 028388) and by the Brain Korea 21 PLUS Project for Medical Science, Yonsei University.

\section{Conflict of interest}

The authors declare no conflicts of interest.

\section{Author contributions}

- C.G.P., H.S.S, H.R.C., and H.Y.N. designed the study and wrote the manuscript.

- H.S.S and H.R.C. performed experiments.

- All authors contributed by proving materials and participating in the analysis.

\section{References}

1. Lambrecht BN, Hammad H. The immunology of asthma. Nat Immunol. 2015; $16: 45-56$

2. Fahy JV. Type 2 inflammation in asthma--present in most, absent in many. Nat Rev Immunol. 2015;15:57-65.

3. Chakir J, Shannon J, Molet S, Fukakusa M, Elias J, Laviolette M, et al. Airway remodeling-associated mediators in moderate to severe asthma: effect of steroids on TGF-beta, IL-11, IL-17, and type I and type III collagen expression. J Allergy Clin Immunol. 2003;111:1293-8.

4. McKinley L, Alcorn JF, Peterson A, Dupont RB, Kapadia S, Logar A, et al. TH17 cells mediate steroid-resistant airway inflammation and airway hyperresponsiveness in mice. J Immunol. 2008;181:4089-97.

5. Bullens DM, Truyen E, Coteur L, Dilissen E, Hellings PW, Dupont LJ, et al. IL-17 mRNA in sputum of asthmatic patients: linking $\mathrm{T}$ cell driven inflammation and granulocytic influx? Respir Res. 2006;7:135.

6. Manni ML, Trudeau JB, Scheller EV, Mandalapu S, Elloso MM, Kolls JK, et al. The complex relationship between inflammation and lung function in severe asthma. Mucosal Immunol. 2014;7:1186-98.

7. Halim TY, Krauss RH, Sun AC, Takei F. Lung natural helper cells are a critical source of Th2 cell-type cytokines in protease allergen-induced airway inflammation. Immunity. 2012;36:451-63.

8. Halim TY, Steer CA, Matha L, Gold MJ, Martinez-Gonzalez I, McNagny $\mathrm{KM}$, et al. Group 2 innate lymphoid cells are critical for the initiation of adaptive $\mathrm{T}$ helper 2 cell-mediated allergic lung inflammation. Immunity. 2014;40:425-35.

9. Kim HY, Lee HJ, Chang YJ, Pichavant M, Shore SA, Fitzgerald KA, et al. Interleukin-17-producing innate lymphoid cells and the NLRP3 inflammasome facilitate obesity-associated airway hyperreactivity. Nat Med. 2014;20:54-61.

10. Warren KJ, Dickinson JD, Nelson AJ, Wyatt TA, Romberger DJ, Poole JA. Ovalbumin-sensitized mice have altered airway inflammation to agriculture organic dust. Respir Res. 2019;20:51.

11. Chapman TJ, Emo JA, Knowlden SA, Rezaee F, Georas SN. Pre-existing tolerance shapes the outcome of mucosal allergen sensitization in a murine model of asthma. J Immunol. 2013;191:4423-30.

12. Chuang YT, Leung K, Chang YJ, DeKruyff RH, Savage PB, Cruse R, et al. A natural killer T-cell subset that protects against airway hyperreactivity. J Allergy Clin Immunol. 2019;143:565-76 e7.

13. Wang X, Hui Y, Zhao L, Hao Y, Guo H, Ren F. Oral administration of Lactobacillus paracasei L9 attenuates PM2.5-induced enhancement of airway hyperresponsiveness and allergic airway response in murine model of asthma. PLoS One. 2017;12:e0171721.

14. Xu L, Sun WJ, Jia AJ, Qiu LL, Xiao B, Mu L, et al. MBD2 regulates differentiation and function of Th17 cells in neutrophils- dominant asthma via HIF-1alpha. J Inflamm (Lond). 2018;15:15.

15. Abdala Valencia H, Loffredo LF, Misharin AV, Berdnikovs S. Phenotypic plasticity and targeting of Siglec-F(high) CD11c(low) eosinophils to the airway in a murine model of asthma. Allergy. 2016;71:267-71.

16. Al-Kouba J, Wilkinson AN, Starkey MR, Rudraraju R, Werder RB, Liu X, et al. Allergen-encoding bone marrow transfer inactivates allergic $\mathrm{T}$ cell responses, alleviating airway inflammation. JCI Insight. 2017;2:

17. Beckert H, Meyer-Martin H, Buhl R, Taube C, Reuter S. The Canonical but Not the Noncanonical Wnt Pathway Inhibits the Development of Allergic Airway Disease. J Immunol. 2018;201:1855-64.

18. Tordesillas L, Goswami R, Benede S, Grishina G, Dunkin D, Jarvinen $\mathrm{KM}$, et al. Skin exposure promotes a Th2-dependent sensitization to peanut allergens. J Clin Invest. 2014;124:4965-75.

19. Shakya AK, Gill HS. A comparative study of microneedle-based cutaneous immunization with other conventional routes to assess feasibility of microneedles for allergy immunotherapy. Vaccine. 2015;33: 4060-4.

20. Hannya N, Ogita-Nakanishi H, Kato R, Ijiri Y, Hayashi T, Tanaka K, et al. The 1st step initiation essential for allergen-specific IgE antibody production upon the 2nd step: Induction of non-specific $\operatorname{IgE}(+)$ small $\mathrm{B}$ cells containing secondly-sensitized allergen-specific ones in mice firstly-sensitized with an allergen. Microbiol Immunol. 2018;62:99-110.

21. Sun W, Xiao B, Jia A, Qiu L, Zeng Q, Liu D, et al. MBD2-mediated Th17 differentiation in severe asthma is associated with impaired SOCS3 expression. Exp Cell Res. 2018;371:196-204.

22. Park Y, Chang YS, Lee SW, Cho SY, Kim YK, Min KU, et al. The enhanced effect of a hexameric deoxyriboguanosine run conjugation to $\mathrm{CpG}$ oligodeoxynucleotides on protection against allergic asthma. J Allergy Clin Immunol. 2001;108:570-6. 
23. Samitas K, Malmhall C, Radinger M, Ramos-Ramirez P, Lu Y, Deak $\mathrm{T}$, et al. Precursor $\mathrm{B}$ Cells Increase in the Lung during Airway Allergic Inflammation: A Role for B Cell-Activating Factor. PLoS One. 2016;11:e0161161.

24. Na HY, Sohn M, Ryu SH, Choi W, In H, Shin HS, et al. Extended Culture of Bone Marrow with Granulocyte Macrophage-Colony Stimulating Factor Generates Immunosuppressive Cells. Immune Netw. 2018;18:e16.

25. Han SM, Na HY, Ham O, Choi W, Sohn M, Ryu SH, et al. TCF4-Targeting miR-124 is Differentially Expressed amongst Dendritic Cell Subsets. Immune Network. 2016;16:61-74.

26. Ryu SH, Na HY, Sohn M, Choi W, In H, Shin HS, et al. Competent antigen-presenting cells are generated from the long-term culture of splenocytes with granulocyte-macrophage colony-stimulating factor. Immunol Lett. 2017;188:96-107.

27. Ryu SH, Na HY, Sohn M, Han SM, Choi W, In $\mathrm{H}$, et al. Reduced expression of granule proteins during extended survival of eosinophils in splenocyte culture with GM-CSF. Immunol Lett. 2016;173:7-20.

28. Dutton EE, Camelo A, Sleeman M, Herbst R, Carlesso G, Belz GT, et al. Characterisation of innate lymphoid cell populations at different sites in mice with defective T cell immunity. Wellcome Open Res. 2017;2:117.

29. Sohn M, Na HY, Ryu SH, Choi W, In H, Shin HS, et al. Two Distinct Subsets Are Identified from the Peritoneal Myeloid Mononuclear Cells Expressing both CD11c and CD115. Immune Netw. 2019;19:e15.

30. Kim HY, Umetsu DT, Dekruyff RH. Innate lymphoid cells in asthma: Will they take your breath away? Eur J Immunol. 2016;46:795-806.
31. Krug N, Madden J, Redington AE, Lackie P, Djukanovic R, Schauer U, et al. T-cell cytokine profile evaluated at the single cell level in BAL and blood in allergic asthma. Am J Respir Cell Mol Biol. 1996;14:319-26.

32. Bloemen K, Verstraelen S, Van Den Heuvel R, Witters H, Nelissen I, Schoeters G. The allergic cascade: review of the most important molecules in the asthmatic lung. Immunol Lett. 2007;113:6-18.

33. Hirose $\mathrm{K}$, Iwata $A$, Tamachi $T$, Nakajima $H$. Allergic airway inflammation: key players beyond the Th2 cell pathway. Immunol Rev. 2017;278:145-61.

34. Qian G, Jiang W, Zou B, Feng J, Cheng X, Gu J, et al. LPS inactivation by a host lipase allows lung epithelial cell sensitization for allergic asthma. J Exp Med. 2018;215:2397-412.

35. Torii I, Shimizu S, Daimon T, Shinohara Y, Kudo T, Sato A, et al. Exposure to High Doses of Lipopolysaccharide during Ovalbumin Sensitization Prevents the Development of Allergic Th2 Responses to a Dietary Antigen. J Toxicol Pathol. 2014;27:205-15.

36. Givens BE, Geary SM, Salem AK. Nanoparticle-based CpG-oligonucleotide therapy for treating allergic asthma. Immunotherapy. 2018;10:595-604.

37. Ding F, Liu B, Zou W, Tian D, Li Q, Dai J, et al. LPS Exposure in Early Life Protects Against Mucus Hypersecretion in Ovalbumin-Induced Asthma by Down-Regulation of the IL-13 and JAK-STAT6 Pathways. Cell Physiol Biochem. 2018;46:1263-74.

38. Bagnasco D, Ferrando M, Varricchi G, Passalacqua G, Canonica GW. A Critical Evaluation of Anti-IL-13 and Anti-IL-4 Strategies in Severe Asthma. International Archives of Allergy and Immunology. 2016;170: 122-31. 